\title{
Effects of calcitriol on oxidative burst, phagocytic function, and leukocyte cytokine production in shelter dogs
}

\author{
Jared A. Jaffey ${ }^{1 *}$, Mariah Bessette ${ }^{1}$, Zenan Tao ${ }^{1}$, Nancy Bradley-Siemens ${ }^{2}$ and Melissa Thompson ${ }^{3}$
}

\begin{abstract}
Background: The active metabolite of vitamin D, calcitriol, has been shown across many different species to augment innate immune responses and dampen aberrant proinflammatory cytokine production. Community acquired infections are common in shelters and consume limited shelter resources, impact adoption rates, and can result in unnecessary euthanasia. Prophylactic oral vitamin D supplementation decreases the incidence and severity of upper and lower respiratory tract infections in humans. Before a clinical trial investigating the clinical benefit of oral vitamin D supplementation in shelter dogs can be pursued, an in vitro study evaluating the immunomodulatory effects of calcitriol in blood from shelter dogs is warranted. Therefore, the objective of this study was to determine if incubation of whole blood obtained from apparently healthy dogs housed in a shelter for $\geq 7$ days with calcitriol would alter granulocyte/monocyte (GM) oxidative burst and phagocytic function as well as pathogen-associated molecular pattern (PAMP)-stimulated leukocyte production of tumor necrosis factor (TNF)-a, and interleukin (IL)-6, and IL-10.

Results: Ten dogs housed in a shelter for $\geq 7$ days were enrolled in a prospective cohort study. Whole blood from these dogs was incubated with calcitriol $\left(10^{-7} \mathrm{M}\right)$ or diluent (control) for $24 \mathrm{~h}$. Subsequent to this incubation, phagocytosis of opsonized-Escherichia coli (E. coli) and E. coli-induced oxidative burst were evaluated via flow cytometry. In addition, leukocyte production of TNF-a, IL-6, and IL-10 were measured using a canine-specific multiplex bead assay. Calcitriol significantly decreased leukocyte TNF-a production $(p=0.009)$ and increased IL-10 production $(p=0.002)$. Tumor necrosis factor-a-to-IL-10 ratio was significantly decreased with calcitriol $(p=0.017)$, while $I L-6$ production as well as GM oxidative burst and phagocytic function were not significantly affected.
\end{abstract}

Conclusions: These data indicate that calcitriol attenuates proinflammatory immune responses without affecting GM oxidative burst or phagocytic function in vitro in whole blood obtained from apparently healthy shelter dogs.

Keywords: Vitamin D, 1,25(oh) ${ }_{2} \mathrm{D}$, Respiratory burst, Phagocytosis, Innate immunity

\footnotetext{
* Correspondence: jjaffe@midwestern.edu

'Department of Specialty Medicine, College of Veterinary Medicine, Midwestern University, 19555 N 59th Ave, Glendale, AZ 85308, USA

Full list of author information is available at the end of the article
} 


\section{Plain English summary}

Vitamin D is an important hormone in musculoskeletal homeostasis but also has an integral role in the immune system. Several studies across many different species highlight that vitamin $\mathrm{D}$ enhances the immune response against infections. In fact, oral vitamin D supplementation has been shown to be well-tolerated and decreases the incidence of acute respiratory tract infections in people. Clinical trials in dogs that evaluate the benefit of oral vitamin D supplementation in the prevention of infections are lacking. Dogs housed in animal shelters commonly develop community acquired infections, which drains limited resources, decreases adoption rates, and can result in unnecessary euthanasia. Oral vitamin D supplementation in shelter dogs could have potential as an affordable and safe means to improve immune function and thus decrease the incidence of infections.

Before a clinical trial can be pursued, a proof of concept in vitro study is warranted to demonstrate that vitamin D has immunologic effects in shelter dogs. In this study we looked at the in vitro effect vitamin D had on the immune function of 10 apparently healthy dogs housed in a shelter. We found that the incubation of blood with vitamin D had significant anti-inflammatory effects without altering the ability of the immune cell to engulf (i.e., phagocytosis) or destroy (i.e., oxidative burst) the bacteria Escherichia coli.

These results indicate that vitamin D has immunologic effects in dogs housed in shelters and provides the needed rationale to pursue a future study that investigates immune function after oral vitamin D supplementation.

\section{Background}

The active metabolite of vitamin $\mathrm{D}$, calcitriol has been shown in many different species, including dogs, to augment innate immune responses [1-11]. Some of these protective effects include an increase in production of antimicrobial peptides, leukocyte reactive nitrogen/oxygen species and phagocytic capacities, and curtails deleterious proinflammatory cytokine responses [1-11]. These immunomodulatory functions highlight the importance of vitamin D to mucosal immunity and corroborates that hypovitaminosis $\mathrm{D}$ in people has been associated with increased susceptibility and severity of illness with inhaled respiratory pathogens [12-15].

Decreased 25-hydroxyvitamin (OH) D concentrations, the primary circulating metabolite of vitamin $\mathrm{D}$, has been identified in many infectious diseases in dogs including leishmaniasis, babesiosis, spirocercosis, blastomycosis, and bacterial sepsis, which suggests that like people, vitamin D could have an important protective role in the canine innate immune response [16-20]. This theory is corroborated by early in vitro studies in healthy and critically ill dogs that demonstrated incubation of blood with calcitriol, decreased leukocyte production of the pro-inflammatory cytokine tumor-necrosis factor (TNF)- $\alpha$ in a concentration dependent manner $[11,21]$. These in vitro results that have highlighted the protective immunomodulatory functions of vitamin $\mathrm{D}$ have translated to yield in vivo benefits with oral supplementation of vitamin $\mathrm{D}$ in people. A recent metaanalysis found that oral supplementation of vitamin $D_{2}$ or vitamin $\mathrm{D}_{3}$ was well tolerated and decreased the incidence of acute respiratory tract infections in people [22]. Similar in vivo studies in dogs are lacking.

Dogs housed in animal shelters represent a unique population that could benefit from oral vitamin D supplementation. The biosynthesis of vitamin D in many species is initiated with exposure to ultraviolet light from the sun and warm ambient temperatures, wherein 7 dehydrocholesterol in the skin is transformed to previtamin $\mathrm{D}_{3}$. Importantly, dogs are not able to synthesize vitamin $D_{3}$ in their skin and thus, are dependent on diet to meet their vitamin $\mathrm{D}$ requirements [23]. It is reasonable to presume that confiscated and rescued dogs could have suboptimal vitamin D concentrations at the time of shelter admission in part because of inadequate nutrition. There is a high incidence of community acquired infections in dogs housed in animal shelters. These infections consume already limited resources, decrease adoption rates, and can even result in unnecessary euthanasia because of herd-health implications. Oral supplementation of vitamin D in dogs at the time of shelter admission could represent a cheap and safe therapy to decrease the incidence, severity of disease, or both of community acquired infections. Before a clinical trial can be pursued, an in vitro study investigating the immunomodulatory effects of vitamin D specifically in shelter dogs is warranted. The in vitro immunomodulatory effects of calcitriol has previously been investigated in healthy dogs [11]; however, dogs housed in animal shelters encounter various factors not accounted for in healthy dogs that could impact immune function including exposure to novel pathogens, stress associated with crowded environment, abrupt change of nutrition, and unknown pre-admission health care and husbandry.

Therefore, the objective of this study was to determine if incubation of whole blood obtained from apparently healthy dogs housed in a shelter for $\geq 7$ days would alter granulocyte/monocyte (GM) oxidative burst and phagocytic function as well as pathogen-associated molecular pattern (PAMP)-stimulated leukocyte production of TNF$\alpha$, interleukin (IL)-6, and IL-10. We hypothesized that incubation of blood obtained from dogs housed in a shelter for $\geq 7$ days would enhance GM oxidative burst and phagocytic function, while also decreasing PAMPstimulated leukocyte production of pro-inflammatory cytokines (i.e., TNF- $\alpha$ and IL-6) with a concomitant increase in the anti-inflammatory cytokine, IL-10. 


\section{Materials and methods}

\section{Animals and selection criteria}

The study was performed as a prospective, in vitro, cohort study. The study protocol was approved by the Midwestern University Animal Care and Use Committee (protocol \# 2932). Ten randomly selected dogs from the Arizona Humane Society were eligible for enrollment in this study. Inclusion criteria consisted of being housed in the shelter for $\geq 7$ days and tested negative for Dirofilaria immitis antigen, and antibodies to Anaplasma phagocytophilum, Anaplasma platys, Ehrlichia canis, Ehrlichia ewingii, and Borrelia burgdorferi C6 peptide using a commercial ELISA-based kit (SNAP 4DX, Plus Test kit, IDEXX Laboratories Inc., Westbrook, ME). Exclusion criteria included detection of $\geq 1$ of the aforementioned pathogens. Furthermore, dogs were excluded if pregnant, lactating, had a surgical procedure performed, or had any illness since shelter admission. Furthermore, dogs were excluded if they had been administered medications, with the exception of routine parasitic prevention or fenbendazole.

\section{Calcitriol}

Calcitriol (Sigma-Aldrich, St. Louis, MO) was dissolved in $75 \%$ ethanol (Sigma-Aldrich, St. Louis, MO) to make a stock solution of calcitriol at $24 \mathrm{nmol} / \mathrm{mL}$ and stored light protected at $4{ }^{\circ} \mathrm{C}$ as previously described [11].

\section{Blood sample collection and calcitriol treatment}

A blood sample $(8 \mathrm{~mL})$ was collected from each dog via jugular venipuncture into tubes containing sodium heparin as an anticoagulant and processed within $1 \mathrm{~h}$. Four mLs of blood was allocated into 2 separate $15 \mathrm{~mL}$ conical tubes for each dog. Blood samples in each conical tube were then diluted 1:2 with RPMI 1640 culture medium (Thermo Fisher Scientific, Carlsbad, CA) containing 200 $\mathrm{U}$ of penicillin/mL and $200 \mathrm{mg}$ of streptomycin $/ \mathrm{mL}$. The blood-RPMI mixture was then incubated with calcitriol (final concentration, $10^{-7} \mathrm{M}$ ) or ethanol negative control substance (final concentration, $6 \times 10^{-2} \mathrm{M}$ ) for $24 \mathrm{~h}$ at $37^{\circ} \mathrm{C}$ in $5 \% \mathrm{CO}_{2}$ in the dark as previously described [11].

\section{Leukocyte cytokine production}

After incubation with calcitriol or control, blood-RPMI mixture was transferred to 96-well plates and stimulated with lipopolysaccharide (LPS) from Escherichia coli O127:B8 (final concentration, $100 \mathrm{ng} / \mathrm{mL}$, Sigma Aldrich, St Louis, MO), lipoteichoic acid (LTA) from Streptococcus faecalis (final concentration, $1 \mu \mathrm{g} / \mathrm{mL}$, Sigma Aldrich, St Louis, MO), or phosphate-buffered saline (PBS) control substance. Plates were incubated for $24 \mathrm{~h}$ at $37^{\circ} \mathrm{C}$ in $5 \% \mathrm{CO}_{2}$ in the dark. Following incubation, plates were centrifuged (400 X g for $7 \mathrm{~min}$ ) at $21^{\circ} \mathrm{C}$ as previously described [11]. The supernatant was collected and stored at $-80{ }^{\circ} \mathrm{C}$ for batch analysis. For analysis, samples were thawed, and then TNF- $\alpha$, IL- 6 , and IL-10 were measured in supernatant with a canine cytokine-specific multiplex bead-based assay (Milliplex MAP canine cytokinechemokine panel, EMD Millipore Corp, Billerica, MA) [11]. The median fluorescence intensity and cytokine concentration in each sample was measured in duplicate with appropriate controls and associated data analysis software (Milliplex Analyst version 5.1, EMD Millipore Corp, Billerica, MA). The lower limit of detection for TNF- $\alpha$, IL-10 was 195 pg/mL and IL-6 was 48.8 pg/mL.

\section{Phagocytosis of $E$. coli}

Phagocytic function of GM was determined with the PhagoTest commercial test kit (Orpegen Pharma, Heidelberg, Germany), validated for use in canines, according to manufacturer's instructions. Briefly, $100 \mu \mathrm{L}$ of blood-RPMI mixture post $24 \mathrm{~h}$ of incubation with calcitriol or control was then incubated in a $37^{\circ} \mathrm{C}$ water bath for $10 \mathrm{~min}$ with $20 \mu \mathrm{L}$ FITC-labeled, opsonized-E. coli bacteria or washing solution (negative control). The samples were placed on ice to arrest phagocytosis and $100 \mu \mathrm{L}$ of quenching solution was added to remove the FITC-fluorescence of surface bound bacteria. The cells were washed, erythrocytes lysed, all cells were washed again, and $200 \mu \mathrm{L}$ of DNA staining solution (R-phycoerythrin) was added to facilitate exclusion of aggregated artifacts of bacteria or cells.

\section{E coli-induced oxidative burst}

Oxidative burst function in GM was determined with a PhagoBurst commercial test kit, validated for use in canines, according to manufacturer's instructions (Orpegen Pharma, Heidelberg, Germany). Briefly, $100 \mu \mathrm{L}$ of bloodRPMI mixture post $24 \mathrm{~h}$ of incubation with calcitriol or control was then incubated with $20 \mu \mathrm{L}$ of opsonized-E. coli or control solution for $10 \mathrm{~min}$ at $37^{\circ} \mathrm{C}$ in a water bath. Next, samples were incubated with $20 \mu \mathrm{L}$ of dihydrorhodamine-123 as a fluorogenic substrate for oxygen intermediates for $10 \mathrm{~min}$ at $37^{\circ} \mathrm{C}$ in a water bath. After cessation of this reaction, erythrocytes were lysed, the cells were washed, and $200 \mu \mathrm{L}$ of DNA staining solution (Rphycoerythrin) was added to facilitate exclusion of aggregated artifacts of bacteria or cells.

\section{Flow cytometry}

Flow cytometry was performed at the Midwestern University College of Veterinary Medicine Immunology Laboratory using a flow cytometer (Guava easyCyte HT, Luminex Corporation, Austin, TX) and associated data analysis software (GuavaSoft 3.2, Luminex Corporation, Austin, TX). A minimum of 20,000 events/sample were recorded. These events were then applied to a forward scatter versus side scatter plot to identify and gate GM 


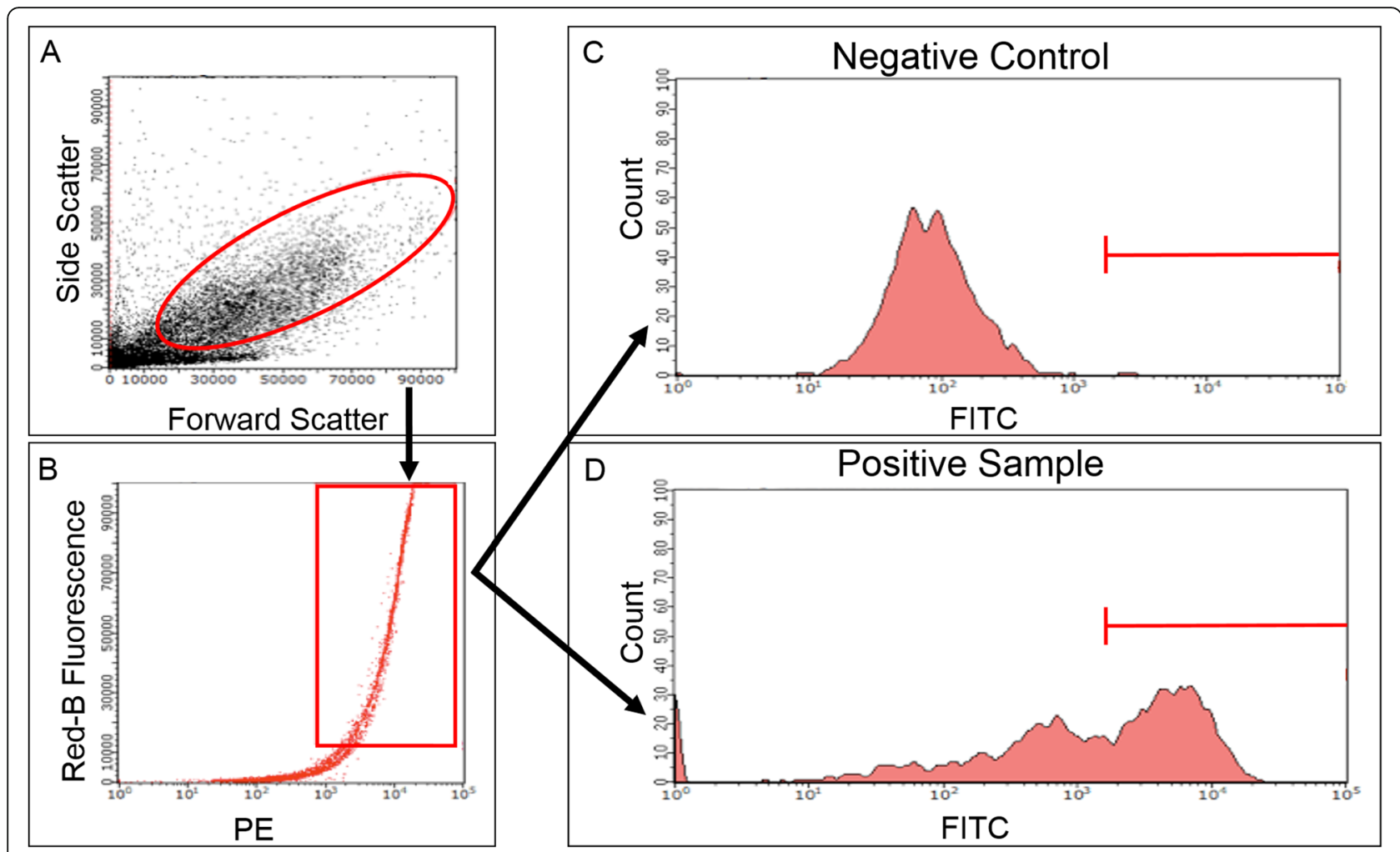

Fig. 1 Gating scheme for flow cytometry of phagocytosis and oxidative burst. Granulocytes and monocytes/macrophages were gated on a forward versus side scatter plot (a), then R-phycoerythrin (PE)-labeled DNA stain was used to exclude aggregates of bacteria or dead cells and positive cells were identified and gated (b). This gate was then applied to appropriate histogram to identify FITC negative (c) and positive cells (d)

populations concurrently (Fig. 1). For assessment of phagocytosis, data were recorded as the percentage of GM cells having internalized FITC-labeled E. coli as well as their mean fluorescent intensity (MFI), a method of quantifying the phagocytosed bacteria per cell. Data for assessment of oxidative burst were recorded as the percentage of GM cells having produced reactive oxygen metabolites and the MFI, the relative robustness of oxidative burst reaction produced per cell.

\section{Statistical analysis}

Statistical analysis was performed by commercial software (SigmaStat, Systat Software Inc). Normality was determined using the Shapiro-Wilk test. Normally distributed data was presented as mean and standard deviation (SD), while data that was not normally distributed was presented as median and interquartile range (IQR). For between-treatment (i.e., calcitriol or control) comparisons (factor 1) for leukocyte production of each TNF- $\alpha$, IL-6, and IL-10 after exposure with LPS, LTA, or PBS (factor 2), a two-way repeated measures analysis of variance (ANOVA) was performed with post hoc Bonferroni $t$-test making pairwise multiple comparisons. Granulocyte/monocyte oxidative burst and phagocytic function data were compared between treatment types (i.e., calcitriol or control) using paired $t$-tests. When the measured TNF- $\alpha$, IL- 6 , and IL-10 concentrations fell below the lower limit of detection data were recorded at the lower limit of detection for statistical purposes. A $p$ value of $<0.05$ was considered significant.

\section{Results}

\section{Animal population}

Sixteen dogs fulfilled the inclusion criteria. Six dogs were excluded because of various health derangements including one each for dehiscence of dental extraction sites, hit by car with lacerations and lameness, canine infectious respiratory disease complex, atopic dermatitis, facial wounds, and diarrhea. Ten dogs were enrolled. Breeds included Chihuahua $(n=4)$, Pit Bull Terrier $(n=3)$, and one each of American Bulldog, Australian Kelpie, and a mixed-breed dog. The average age was 3.4 years (SD, 1.8). There were 4 intact males, 3 spayed females, and 3 neutered males. The median weight and duration of time spent in shelter were $9.5 \mathrm{~kg}$ (IQR; 3.9-24.2) and 10 days (IQR; 9.8-12.0).

\section{Leukocyte cytokine responses}

There was a significant between-treatment difference in leukocyte production of TNF- $\alpha(p=0.009)$ but not IL-6 $(p=0.12)$, irrespective of type of PAMP-exposure (i.e., 


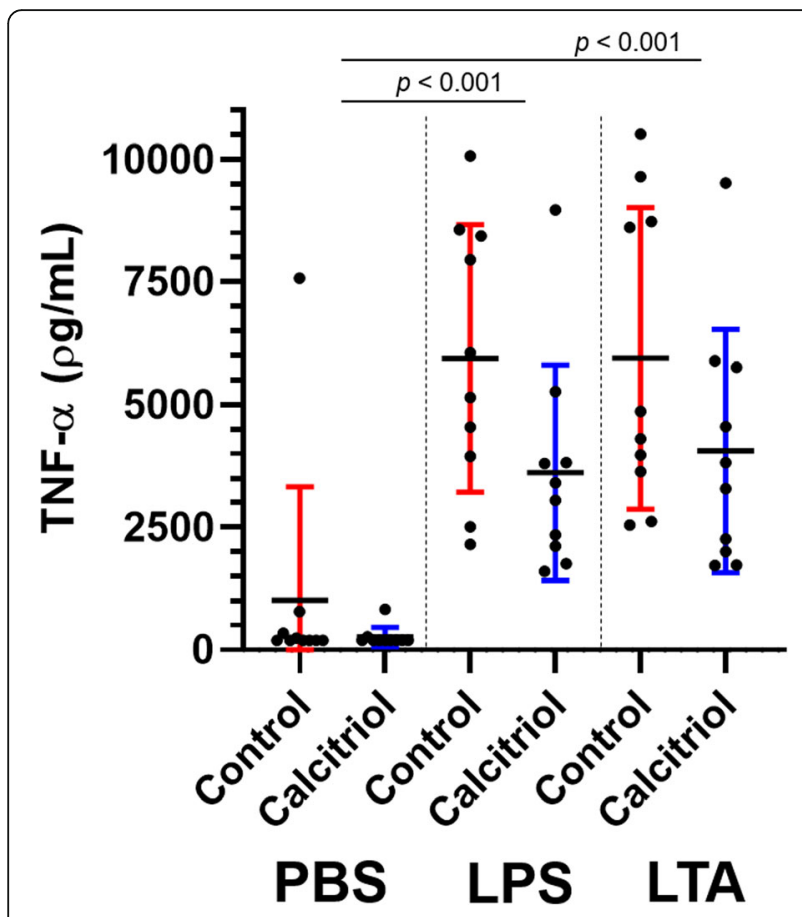

Fig. 2 Two-way repeated measured analysis of variance comparing leukocyte production of tumor necrosis factor (TNF)-a between blood samples incubated in calcitriol (blue lines, $n=10$ ) and control (red lines, $n=10$ ) with subsequent exposure to phosphate buffered saline (PBS), lipopolysaccharide (LPS), or lipoteichoic acid (LTA). The black lines represent the mean, bars the SD, and the black circles are individual dog data. There were significant between-treatment $(p=$ 0.009) and between-pathogen associated molecular pattern (PAMP)exposure $(p<0.001)$ differences. There was not a treatment-by-PAMP exposure interaction $(p=0.08)$

LPS, LTA, PBS; Figs. 2, 3). Post hoc tests revealed that leukocyte production of TNF- $\alpha$ significantly decreased when cells were incubated with calcitriol compared to control (Fig. 2). A significant between-PAMP-exposure difference in leukocyte production of TNF- $\alpha(p<0.001)$ and IL-6 $(p<0.001)$, irrespective of cell treatment (i.e., calcitriol or control) was identified (Figs. 2, 3). As expected, post hoc tests revealed that LPS- and LTAexposed leukocytes produced significantly greater TNF- $\alpha$ and IL- 6 than leukocytes exposed to PBS $(p<$ 0.001 ; Figs. 2,3$)$. There was a significant treatmentby-PAMP-exposure interaction for leukocyte production of IL-10 ( $p=0.01$; Fig. 4). Therefore, betweentreatment and between-PAMP-exposure differences for IL-10 could not be measured because of this interaction. Post hoc tests revealed that cells incubated with calcitriol produced significantly greater IL10 compared with control when exposed with LTA $(p=0.002$; Fig. 4). Further, these post hoc tests showed that cells incubated with calcitriol $(p<0.001)$ or control $(p=0.03)$ produced significantly greater IL10 when exposed with LTA compared to PBS (Fig. 4).

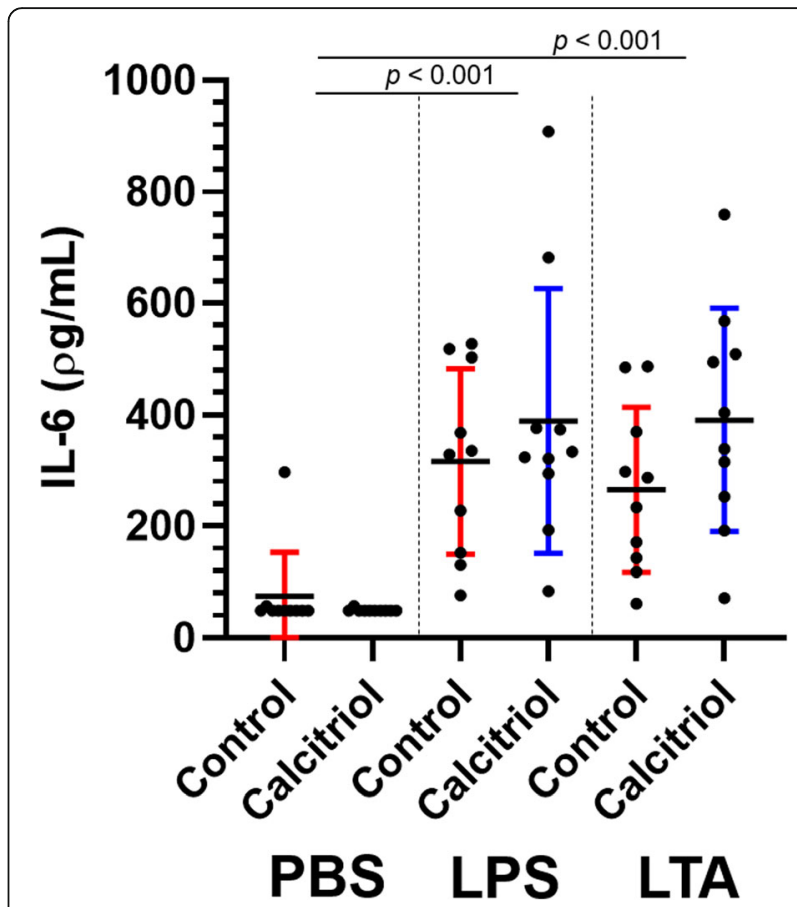

Fig. 3 Two-way repeated measured analysis of variance comparing leukocyte production of interleukin (IL)-6 between blood samples incubated in calcitriol (blue lines, $n=10$ ) and control (red lines, $n=$ 10) with subsequent exposure to phosphate buffered saline (PBS), lipopolysaccharide (LPS), or lipoteichoic acid (LTA). The black lines represent the mean, bars the SD, and the black circles are individual dog data. There was a significant between-pathogen associated molecular pattern (PAMP)-exposure difference $(p<0.001)$, but not a between-treatment difference $(p=0.12)$. There was not a treatmentby-PAMP exposure interaction $(p=0.07)$

\section{TNF-a-to-IL-10 ratio}

A significant treatment-by-PAMP exposure interaction was identified for TNF- $\alpha$-to-IL-10 ratio precluding analysis of between-treatment and between-PAMP exposure differences $(p=0.017$; Fig. 5). Post hoc tests revealed that incubation of cells with calcitriol resulted in a significant decrease in TNF- $\alpha$-to-IL-10 ratio compared to control, when cells were exposed to LPS $(p<0.001)$ or LTA $(p=0.004$; Fig.5). In addition, as expected, LPS-exposure in cells yielded a significantly greater TNF- $\alpha$-to-IL-10 ratio than PBS-exposed cells incubated with control ( $p=0.002$; Fig. 5).

\section{Phagocytosis and oxidative burst}

There was not a significant difference in the percentage of GM that phagocytized opsonized-E. coli or number of phagocytized opsonized-E. coli per cell between blood samples incubated with calcitriol or control (Fig. 6a,b). Technical difficulties precluded the inclusion of phagocytosis results from 1 dog. Likewise, a significant difference in the percentage of GM that had performed E.coliinduced oxidative burst as well as the E.coli-induced oxidative burst per cell was not identified (Fig. 6c,d). 


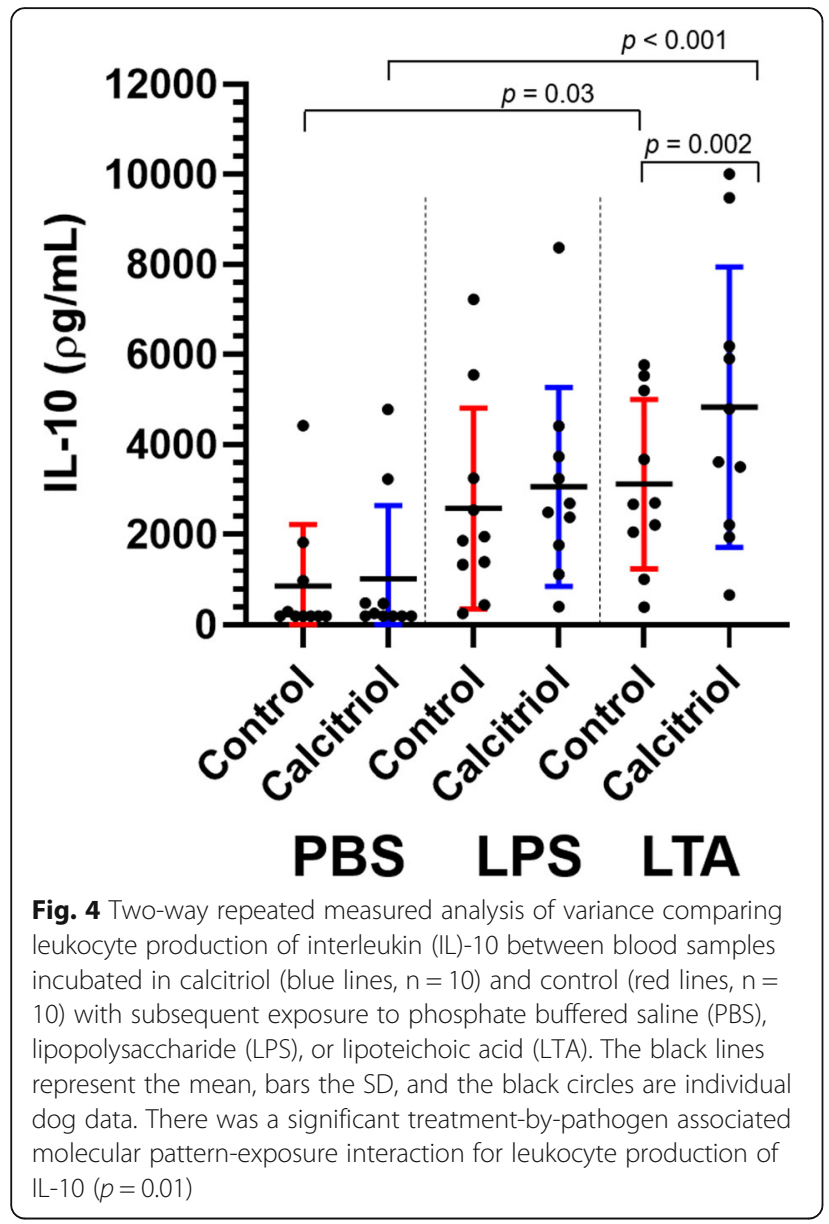

\section{Discussion}

In the present study, we evaluated the effects of calcitriol on leukocyte cytokine production and GM E. coliinduced-oxidative burst and phagocytosis of opsonizedE. coli in blood samples obtained from apparently healthy dogs housed in a shelter for $\geq 7$ days. Incubation of blood with calcitriol resulted in a decreased leukocyte production of TNF- $\alpha$, irrespective of the type of PAMPexposure. Leukocytes incubated with calcitriol produced greater IL-10 with subsequent LTA-exposure. There was not a between-treatment (i.e., incubation with calcitriol or control) difference in leukocyte production of IL-6. The inflammatory milieu when evaluated in the context of TNF- $\alpha$-to-IL-10 ratio, was shifted to an antiinflammatory phenotype when blood was incubated with calcitriol when exposed to LPS and LTA. Calcitriol did not affect $E$. coli-induced oxidative burst or phagocytosis of opsonized-E. coli.

Incubation of blood with calcitriol resulted in a decrease in leukocyte production of TNF- $\alpha$, irrespective of the type of PAMP-exposure. This finding supports our hypothesis and corroborates results from previous studies that demonstrated calcitriol decreased leukocyte

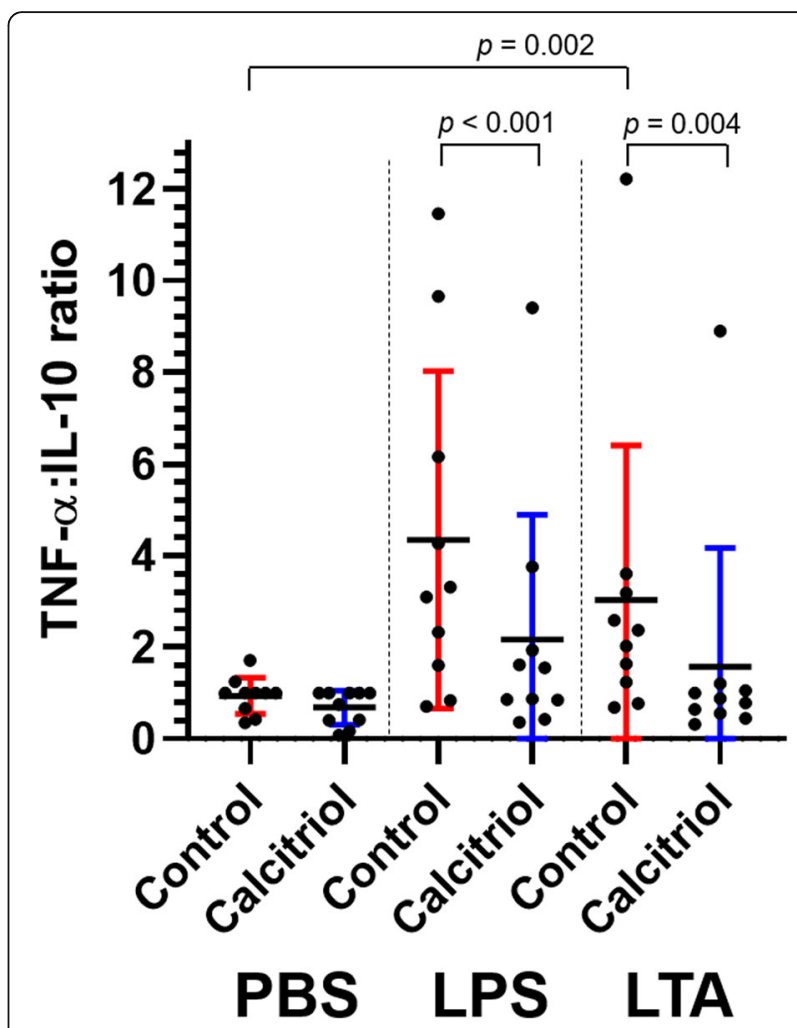

Fig. 5 Two-way repeated measured analysis of variance comparing tumor necrosis factor (TNF)-a-to-interleukin (IL)-10 ratios between blood samples incubated in calcitriol (blue lines, $n=10$ ) and control (red lines, $n=10$ ) with subsequent exposure to phosphate buffered saline (PBS), lipopolysaccharide (LPS), or lipoteichoic acid (LTA). The black lines represent the mean, bars the SD, and the black circles are individual dog data. There was a significant treatment-by-pathogen associated molecular pattern-exposure interaction for TNF-a-to-IL-10 ratio $(p=0.017)$

production of TNF- $\alpha$ in blood obtained from healthy and critically-ill dogs [11, 21]. The molecular etiology for calcitriol induced modulation of TNF- $\alpha$ production in dogs is largely unknown. However, there are multiple proposed mechanisms in humans that are postulated to explain this process. Ligation of toll-like receptors (TLR) with their respective PAMP initiates a cascade of signaling pathways that result in the induction of proinflammatory cytokines (e.g., TNF- $\alpha$, IL-6, IL-1). In humans and rodents, studies have illustrated that calcitriol decreases monocyte expression of TLR-2 and TLR-4 resulting in a relative decrease in the quantity of available receptors to propagate inflammation [24, 25]. A small in vitro pilot study in dogs did not find that calcitriol had a significant effect on GM TLR-4 expression [11]. Calcitriol also suppresses the activation and signaling of proinflammatory pathways including nuclear factor (NF)-kB and mitogen-activated protein kinase (MAPK) [26, 27]. Interestingly, calcitriol did not decrease leukocyte production of IL-6 in this study. The 

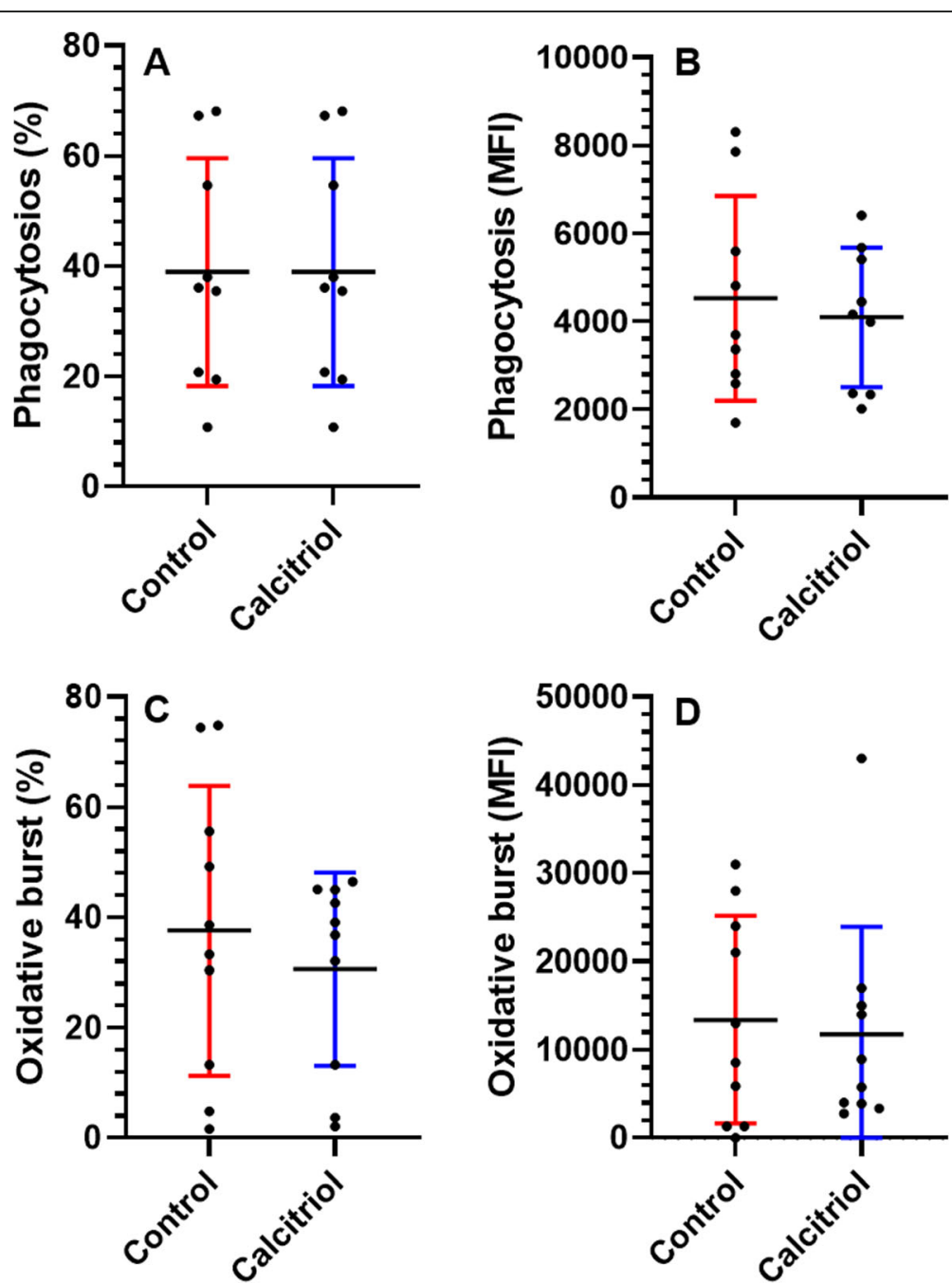

Fig. 6 Two-tailed paired t-test comparing the percentage of granulocytes/monocytes (GM) that phagocytized opsonized-E. coli $(A, n=9)$ and performed E. coli-induced oxidative burst $(C, n=10)$ as well as the number of phagocytized opsonized-E. coli per cell $(B, n=10)$ and the intensity of $E$. coli-induced oxidative burst per cell $(D, n=10)$ in samples incubated with calcitriol (blue lines) or control (red lines). The black lines represent the mean, bars the SD, and the black circles are individual dog data

reason for this lack of response is unknown and could be related to the small study population. This proinflammatory cytokine is influenced by the same molecular pathways that mediate production of TNF- $\alpha$ (i.e., NFkB and MAPK). Additional studies with larger populations are needed to better understand the effect calcitriol has on leukocyte production of IL-6 in dogs.

During infection, the host innate immune response generated against pathogens can be unbalanced and potentially detrimental. Tumor necrosis factor- $\alpha$ has long been implicated in the development of immunopathology and tissue injury [28]. The deleterious effects of TNF- $\alpha$ are conserved across various species, including dogs. To the authors' knowledge, the role of proinflammatory cytokines including TNF- $\alpha$ on morbidity and mortality of shelter and non-shelter dogs with spontaneous community-acquired infections has not been investigated. However, studies in humans illustrate that many community-acquired upper and lower respiratory infections are associated with exaggerated production of proinflammatory cytokines including TNF- $\alpha$ [29]. Moreover, studies have demonstrated that TNF- $\alpha$ activity is associated with the severity of disease and prolonged production of TNF- $\alpha$ exacerbates illness [30-32]. Future clinical trials are needed to investigate if prophylactic oral administration of vitamin $\mathrm{D}$ to shelter dogs dampens aberrant production of TNF- $\alpha$.

The incubation of leukocytes with calcitriol significantly increased production of IL-10 when exposed to LTA, but not LPS. These results indicate that calcitriol could partially abrogate aberrant host inflammatory response when exposed to gram positive bacteria. The 
immunomodulatory effect that calcitriol has on PAMPexposed leukocyte IL-10 production has a great deal of intra- and interspecies variability [33-36]. A previous small pilot study in dogs determined that calcitriol did not increase leukocyte production of IL-10 when exposed to LPS or LTA [11]. A subsequent series of in vitro studies showed that priming of cells (e.g., endotoxin or endogenous inflammatory mediators) before incubation with calcitriol could be one factor that is needed for an increase in leukocyte production of IL-10 with subsequent LPS-exposure [37]. The reason for a potential contrasting production capacity of IL-10 in cells incubated with calcitriol when exposed to LPS versus LTA as was seen in this study is unknown. In humans, calcitriol bound to its receptor directly increases expression of IL-10 independent of the pathways mediated by TLR/PAMP ligation [38].

Calcitriol significantly decreased the TNF- $\alpha$-to-IL-10 ratio with post-incubation exposure of LPS and LTA. Tumor necrosis factor- $\alpha$-to-IL-10 ratio is often used to investigate shifts in host inflammatory phenotype [39]. Our results indicate that pre-incubation of leukocytes obtained from shelter dogs with calcitriol dampens the proinflammatory response to post-incubation PAMPstimulation. It is also important to consider that a decreased TNF-a-to-IL-10 ratio is not uniformly an immunologic advantage. This tolerogenic shift dampens potentially deleterious exaggerated inflammation, but this could also coincide with a compromised immune response to pathogens. Community-acquired infections, especially community acquired respiratory disease complex has a high prevalence in shelters [40]. The highly infectious nature of the complex represents a great burden on shelter resources. Prophylactic oral supplementation of vitamin $\mathrm{D}$ has been shown to decrease the incidence and severity of respiratory infections in humans $[22,41]$. Future clinical trials are needed to investigate if prophylactic oral administration of vitamin $D$ to shelter dogs' decreases TNF- $\alpha$-to-IL-10 ratio in vivo and if this translates to decreased incidence, morbidity, or both of community acquired infections.

In contrast to human monocytes/macrophages, incubation of cells with calcitriol did not significantly affect leukocyte phagocytic or oxidative burst functions in our apparently healthy shelter dogs $[6,10,42-44]$. Our study evaluated the effect of calcitriol on GM phagocytosis and oxidative burst associated with E. coli. Many of the aforementioned studies in humans specifically investigated the in vitro effect that calcitriol had on monocyte/ macrophage immune responses to Mycobacterium tuberculosis. Pathogen recognition, phagocytosis, and subsequent pathogen killing is a selective process, requiring specific interactions between host immune cell receptors and pathogens. Mycobacterium tuberculosis interacts with various different TLRs including 2, 4, 8, and 9 found predominately with macrophages [45]. In contrast, E. coli interacts with most cells in the innate immune system via TLR-4 alone. While most human studies highlight a uniformly protective effect of calcitriol against Mycobacterium tuberculosis, there are conflicting reports on the benefit of calcitriol against $E$. coli $[46,47]$. Therefore, it is possible that calcitriol has differential immune responses dependent on the type of pathogen encountered by the host. In addition, this study utilized a single concentration of calcitriol (i.e., $10^{-7} \mathrm{M}$ ) and incubation time period (i.e., $24 \mathrm{~h}$ ). Additional studies that utilize various calcitriol concentrations and incubation times are needed.

Our study had several limitations. We utilized whole blood cultures to evaluate various effects on immune responses. This global approach allowed us to investigate the broad and diverse effects of calcitriol in a manner more relatable to in vivo microenvironments and immune interactions. This study used E. coli as the model micro-organism in its in vitro evaluation of GM phagocytic and oxidative burst capacities. This approach limits the interpretation of these results to pathogens that ligate TLR-4 (i.e., a gram-negative bacterium like $E$. coli). These results could be representative of the canine innate immune response to Bordetella bronchiseptica, a common gram-negative CIRD pathogen that ligates TLR-4; however, they do not reflect the immune response to other bacterial and viral pathogens that utilize other immunologic pathways. Additional studies are needed to explore the in vitro effect of calcitriol with micro-organisms that utilize a broader array of leukocyte recognition pathways. In addition, the short-term incubation of whole blood with calcitriol may not replicate the in vivo immunomodulatory effects of oral supplementation with vitamin D in dogs. Eventual in vivo clinical trials are needed to further explore this. Importantly, the results from this in vitro study should not be extrapolated into in vivo clinical situations. We investigated the effect of calcitriol on specific immune functions. In other words, these tests were performed in a vacuum and the net effect could be different and potentially detrimental to clinical patients. Future in vivo studies are needed before vitamin $\mathrm{D}$ can be applied as a therapeutic intervention in dogs. Another limitation is that we enrolled only apparently healthy dogs. Calcitriol could have a different effect on shelter dogs that were systemically ill because they would be expected to have decreased serum $25(\mathrm{OH}) \mathrm{D}$ concentrations compared to healthy dogs [20]. Humans that are vitamin $\mathrm{D}$ deficient experience the most clinical benefit when supplemented with oral vitamin D [22].

\section{Conclusions}

These data indicate that calcitriol is capable of shifting the phenotype of leukocytes obtained from shelter dogs 
from proinflammatory to anti-inflammatory. Calcitriol did not affect GM phagocytosis of opsonized-E. coli or E. coli-induced oxidative burst. Future in vitro and in vivo studies in healthy and sick shelter dogs are needed to better understand the potential therapeutic benefit of oral vitamin D supplementation.

\section{Acknowledgements}

The authors would like to thank Paige Hunsinger, Heather Hotchkiss, and Sasha Willis for their technical expertise.

\section{Authors' contributions}

JAJ was responsible for study design, data analysis, and manuscript preparation. MB was responsible for recruitment, data acquisition, and manuscript preparation. ZT was responsible for data acquisition/analysis and manuscript preparation. NSB was responsible for recruitment, data acquisition, and manuscript preparation. MT was responsible for recruitment, data acquisition, and manuscript preparation. The author(s) read and approved the final manuscript.

\section{Funding}

Maddie's Fund provided partial financial support of this project through the Maddies Idea Lab Grant.

\section{Availability of data and materials}

The datasets used and/or analyzed during the current study are available from the corresponding author on reasonable request.

\section{Ethics approval and consent to participate}

The study protocol was approved by the Midwestern University Animal Care and Use Committee (protocol \# 2932).

\section{Consent for publication}

Not applicable.

\section{Competing interests}

The authors have no financial or personal relationships that could inappropriately influence or bias the content of the paper.

\section{Author details}

'Department of Specialty Medicine, College of Veterinary Medicine, Midwestern University, 19555 N 59th Ave, Glendale, AZ 85308, USA ${ }^{2}$ Department of Pathology and Population Medicine, College of Veterinary Medicine, Midwestern University, 19555 N 59th Ave, Glendale, AZ 85308,

USA. ${ }^{3}$ Arizona Humane Society, 9226 N 13th Ave, Phoenix, AZ 85021, USA.

Received: 24 May 2020 Accepted: 4 August 2020

Published online: 14 August 2020

\section{References}

1. Rockett KA, Brookes R, Udalova I, Vidal V, Hill AVS, et al. Kwiatkowski, D. 1,25dihydroxyvitamin D3 induces nitric oxide synthase and suppresses growth of mycobacterium tuberculosis in a human macrophage-like cell line. Infect Immun. 1998;66:5314-21.

2. Sly LM, Lopez M, Nauseef WM, Reiner NE. 1alpha,25-dihydroxyvitamin D3induced monocyte antimycobacterial activity is regulated by phosphatidylinositol 3-kinase and mediated by the NADPH-dependent phagocyte oxidase. J Biol Chem. 2001;276:35482-93.

3. Chandra G, Selvaraj P, Jawahar MS, Banurekha W, Narayanan PR. Effect of vitamin D3 on phagocytic potential of macrophages with live mycobacterium tuberculosis and lymphoproliferative response in pulmonary tuberculosis. J Clin Immunol. 2004;24:249-57.

4. Martineau AR, Wilkinson KA, Newton SM, Floto RA, Norman AW, et al. IFN(gamma)- and TNF-independent vitamin D-inducible human suppression of mycobacteria: the role of cathelicidin II-37. J Immunol. 2007;178:7190-8.

5. Tiosano D, Wildbaum G, Gepstein-Verbitsky O, Weisman Y, Karin N, et al. The role of vitamin $D$ receptor in innate and adaptive immunity: a study in hereditary vitamin D-resistant rickets patients. J Clin Endocrinol Metab. 2013; 98:1685-93.
6. Motlagh BM, Ahangaran NA, Froushani SMA. Calcitriol modulates the effects of bone marrow-derived mesenchymal stem cells on macrophage functions. Iran J Med Sci. 2015;18:672-6.

7. Lin Z, Li W. The roles of vitamin D and its analogs in inflammatory diseases. Curr Top Med Chem. 2016;16:1242-61.

8. Rodriguez-Lecompte JC, Yitbarek A, Cuperus T, Echeverry H, van Dijk A. The immunomodulatory effect of vitamin $D$ in chickens is dose-dependent and influenced by calcium and phosphorus levels. Poult Sci. 2016;95:2547-56.

9. Vieira-Neto A, Lima IRP, Lopes F Jr, Lopera C, Zimpel R, Sinedino LDP, Jeong KC, Galvao K, Thatcher WW, Nelson CD, et al. Use of calcitriol to maintain postpartum blood calcium and improve immune function in dairy cows. J Dairy Sci. 2017;100:5805-23.

10. Garcia-Barragan A, Gutierrez-Pabello JA, Alfonseca-Silva E. Calcitriol increases nitric oxide production and modulates microbicidal capacity against Mycobacterium bovis in bovine macrophages. Comp Immunol Microbiol Infect Dis. 2018;59:17-23.

11. Jaffey JA, Amorim J, DeClue AE. Effects of calcitriol on phagocytic function, toll-like receptor 4 expression, and cytokine production in canine leukocytes. Am J Vet Res. 2018:79:1064-70.

12. Laaksi I, Ruohola JP, Tuohimaa P, Auvinen A, Haataja R, et al. An association of serum vitamin $D$ concentrations $<40 \mathrm{nmol} / \mathrm{L}$ with acute respiratory tract infection in young Finnish men. Am J Clin Nutr. 2007:86:714-7.

13. Sabetta JR, DePetrillo P, Cipriani RJ, Smardin J, Burns LA, et al. Serum 25hydroxyvitamin $\mathrm{D}$ and the incidence of acute vital respiratory tract infection in healthy adults. PLoS One. 2010;5:e1 1088.

14. Mamani M, Muceli N, Ghasemi Basir HR, Vasheghani M, Poorolajal J. Association between serum concentration of 25-hydroxyvitamin $D$ and community-acquired pneumonia: a case-control study. Int J Gen Med. 2017; 10:423-9.

15. Li W, Cheng X, Guo L, Li H, Sun C, et al. Association between serum 25hydroxyvitamin $\mathrm{D}$ concentration and pulmonary infection in children. Medicine. 2018:97:e9060

16. O'Brien MA, McMichael MA, Le Boedec K. 25-hydroxyvitmian D concentrations in dogs with naturally acquired blastomycosis. J Vet Intern Med. 2018;32:1684-91

17. Rosa CT, Schoeman JP, Berry JL, Mellanby RJ, Dvir E. Hypovitaminosis D in dogs with spirocercosis. J Vet Intern Med. 2013;27:1159-64.

18. Rodriguez-Cortes A, Martori C, Martinez-Florez A, Clop A, Amills M, et al. Canine leishmaniasis progression is associated with vitamin d deficiency. Sci Rep. 2017;7:3346.

19. Dvir E, Rosa C, Handel I, Mellanby RJ, Schoeman JP. Vitamin D status in dogs with babesiosis. Onderstepoort J Vet Res. 2019;86:e1-5.

20. Jaffey JA, Backus RC, McDaniel KM, DeClue AE. Serum vitamin D concentrations in hospitalized critically ill dogs. PLoS One. 2018;13: e0194062.

21. Jaffey JA, Amorim J, DeClue AE. Effect of calcitriol on in vitro whole blood cytokine production in critically ill dogs. Vet J. 2018;236:31-6.

22. Martineau AR, Jolliffe DA, Hooper RL, Greenberg L, Aloia JF, et al. Vitamin D supplementation to prevent acute respiratory tract infections: systematic review and meta-analysis of individual participant data. BMJ. 2017;356:6583.

23. How KL, Hazewinkel HA, Mol JA. Dietary vitamin D dependence of cat and dog due to inadequate cutaneous synthesis of vitamin D. Gen Comp Endocrinol. 1994:96:12-8.

24. Sadeghi K, Wessner B, Laggner U, Ploder M, Tamandl D, et al. Vitamin D3 down-regulates monocyte TLR expression and triggers hyporesponsiveness to pathogen-associated molecular patterns. Eur J Immunol. 2006;36:361-70.

25. Chen $Y$, Liu W, Sun T, Huang $Y$, Wang $Y$, et al. 1,25-dihydroxyvitamin D promotes negative feedback regulation of TLR signaling via targeting microRNA-155-SOCS1 in macrophages. J Immunol. 2013;190:3687-95.

26. Stio M, Martinesi M, Bruni S, Treves C, Mathieu C, et al. Vitamin D analogue TX 527 blocks NF-kB activation in peripheral blood mononuclear cells of patients with Chron's disease. J Steroid Biochem Mol Biol. 2007;103:51-60.

27. Zhang $Y$, Leung DYM, Richers $B N$, Liu $Y$, Remigio $L K$, et al. Vitamin $D$ inhibits monocyte /macrophage proinflammatory cytokine production by targeting MAPK phosphatase-1. J Immunol. 2012;188:2127-35.

28. Bekker LG, Moreira AL, Bergtold A, Freeman S, Ryffel B, et al. Immunopathologic effects of tumor necrosis factor alpha in murine mycobacterial infection are dose dependent. Infect Immun. 2000:68:695461.

29. Glezen P, Denny FW. Epidemiology of acute lower airway disease in children. N Engl J Med. 1973;288:498. 
30. Matsuda K, Tsutsumi H, Okamoto Y, Chiba C. Development of interleukin 6 and tumor necrosis factor alpha activity in nasopharyngeal secretions of infants and children during infection with respiratory syncytial virus. Clin Diagn Lab Immunol. 1995:2:322-4.

31. Noah TL, Henderson FW, Wortman IA, Devlin RB, Handy J, et al. Nasal cytokine production in vital acute upper respiratory infection of childhood. J Infect Dis. 1995;171:584-92.

32. Rutigliano JA, Graham BS. Prolonged production of TNF-a exacerbates illness during respiratory syncytial virus infection. J Immunol. 2004;173:340817.

33. He CS, Fraser WD, Gleeson M. Influence of vitamin D metabolites on plasma cytokine concentrations in endurance sport athletes and on multiantigen stimulated cytokine production by whole blood and peripheral blood mononuclear cell cultures. Int Sch Res Notices. 2014;820524:1-9.

34. Morris A, Selvaraj RK. In vitro 25-hydroxycholecalciferol treatment of lipopolysaccharide-stimulated chicken macrophages increases nitric oxide production and mRNA of interleukin-1B and 10. Vet Immunol Immunopathol. 2014;161:265-70.

35. Niino M, Fukazawa T, Miyazaki Y, Takahashi E, Minami N, et al. Suppression of IL-10 production by calcitriol in patients with multiple sclerosis. Neuroimmunol. 2014;270:86-94.

36. Xu QA, Li ZF, Zhang P, Cao LH, Fan MW. Effects of 1,25-dihydroxyvitamin D3 on macrophage cytokine secretion stimulated by Porphyromonas gingivalis. Jpn J Infect Dis. 2016;69:482-7.

37. Jaffey JA, Amorim J, DeClue AE. Effects of calcitriol on apoptosis, toll-like receptor 4 expression, and cytokine production on endotoxin-primed canine leukocytes. Am J Vet Res. 2018;79:1071-8.

38. Heine G, Niesner U, Chang HD, Steinmeyer A, Zugel U, et al. 1,25dihydroxyvitamin D3 promotes IL-10 production in human B cells. Eur J Immunol. 2008;38:2210-8.

39. Goswami B, Rajappa M, Mallika V, Shukla DK, et al. TNF-a/IL-10 ratio and Creactive protein as markers of the inflammatory response in CAD-prone north Indian patients with acute myocardial infarction. Clin Chim Acta. 2009; 408:14-8.

40. Protopopova A, Hall NJ, Brown KM, Andrukonis AS, Hekman JP. Behavioral predictors of subsequent respiratory illness signs in dogs admitted to an animal shelter. PLoS One. 2019;14:e0224252.

41. Jung HC, Seo MW, Lee S, Kim SW, Song JK. Vitamin D3 supplementation reduces the symptoms of upper respiratory tract infection during winter training in vitamin D-insufficiency taekwondo athletes: a randomized controlled trial. Int J Environ Res Public Health. 2018;15:E2003.

42. Levy R, Malech HL. Effect of 1,25-dihydroxyvitamin D3, lipopolysaccharide, or lipoteichoic acid on the expression of NADPH oxidase components in cultured human monocytes. J Immunol. 1991;147:3066-71.

43. Tokuda N, Levy RB. 1,25-Dihydroxyvitamin D3 stimulates phagocytosis but suppresses HLA-DR and CD13 antigen expression in human mononuclear phagocytes. Exp Biol Med. 1996;211:244-50.

44. Nouari W, Ysmail-Dahlouk L, Aribi M. Vitamin D3 enhances bactericidal activity of macrophages against Pseudomonas aeruginosa. Int Immunopharmacol. 2016;30:94-101.

45. Faridghohar $\mathrm{M}$, Nikoueinejad $\mathrm{H}$. New findings of toll-like receptors involved in Mycobacterium tuberculosis infection. Pathog Glob Health. 2017;111:25664.

46. Xu H, Soruri A, Gieseler RK, Peters JH. 1,25-dihydroxyvitamin D3 exerts opposing effects to IL-4 on MHC-class II antigen expression, accessory activity, and phagocytosis of human monocytes. Scand J Immunol. 1993;38: 535-40.

47. Spittler A, Willheim M, Leutmezer F, Ohler R, Krugluger W, et al. Effects of 1a,25-dihydroxyvitamin D3 and cytokines on the expression of MHC antigens, complement receptors and other antigens on human blood monocytes and U937 cells: role in cell differentiation, activation and phagocytosis. Immunology. 1997;90:286-93.

\section{Publisher's Note}

Springer Nature remains neutral with regard to jurisdictional claims in published maps and institutional affiliations.

\section{Ready to submit your research? Choose BMC and benefit from:}

- fast, convenient online submission

- thorough peer review by experienced researchers in your field

- rapid publication on acceptance

- support for research data, including large and complex data types

- gold Open Access which fosters wider collaboration and increased citations

- maximum visibility for your research: over $100 \mathrm{M}$ website views per year

At BMC, research is always in progress.

Learn more biomedcentral.com/submissions 